\title{
CARACTERIZAÇÃO DA PRODUÇÃO CIENTÍFICA SOBRE DOENÇAS SEXUALMENTE TRANSMISSIVEIS E HIV/AIDS PUBLICADOS EM PERIÓDICOS DE ENFERMAGEM DO BRASIL
}

\author{
CHARACTERISTICS OF SCIENTIFIC PRODUCTION ABOUT SEXUALLY \\ TRANSMITTED DISEASES AND HIV/AIDS- PUBLISHED IN BRAZILIAN \\ NURSING JOURNAL
}

\section{CARACTERIZACIÓN DE PRODUCCION CIENTÍFICA SOBRE ENFERMEDADES SEXUALMENTE TRASMISIBLES Y SIDA PUBLICADOS EN PERIÓDICOS DE ENFERMERIAA DE BRASIL}

Renata Karina Reis* Elucir Gir**

Reis RK, Gir E. Caracterização da produção científica sobre doenças sexualmente transmissiveis e HIV/AIDS publicados em periódicos de enfermagem doBrasil. Rev Esc Enferm USP 2002; 36(4): 376-85.

\section{RESUMO}

O controle das DST/aids representa desafio para a humanidade, considerando seu impacto biopsicosocial. Diante desta realidade, realizou-se um estudo exploratório, descritivo e documental, com os objetivos de caracterizar a produção científica sobre DST em 14 periódicos de enfermagem do Brasil, publicada no periodo de 1933 a 1999. A amostra constituiu-se de 73 publicações, sendo 55 referentes à temática HIV/aids e 18 sobre as demais DST. Os dados evidenciam que os autores, em sua maioria, são enfermeiros, atuam na área de docência, são procedentes do Estado de São Paulo, e têm privilegiado a temática HIV/aids em detrimento das outras DST, sendo sua produção científica escassa e descontinua.

PALAVRAS-CHAVE: Doenças sexualmente transmissiveis. Sindrome de imunodeficiência adquirida. Publicações.

\begin{abstract}
STD/Aids control represents a challenge to humanity due to its biopychosocial impact. In view of this reality, an exploratory, descriptive and documental study was conducted with the purpose to characterize the scientific production on STD published in 14 Brazilian nursing journals from 1933 to 1999 . The sample consisted of 73 publications of which 55 concerned HIV/Aids and 18 were related to other STD. The data showed that most of the authors were faculty nurses from São Paulo Estate who have privileged HIV/Aids in detriment of other STD and that their scientific production was scarse and discontinuos.
\end{abstract}

KEYWORDS: Sexually transmitted diseases. Acquired immunodeficiency syndrome. Publications.

\section{RESUMEN}

El control de las Enfermedades Sexualmente Transmisibles/SIDA representa desafio para la humanidad, considerando su impacto biopsicoscial. Delante de esa realidad, fue realizado un estudio exploratório, descriptivo y documental, con los objetivos de caracterizar la produccion científica sobre el SIDA en 14 periódicos de enfermería de Brasil, publicada en el periodo entre 1933 y 1999. La muestra fue constituida de 73 publicaciones, sindo 55 referientes a la temática HIV/SIDA y 18 sobre otras enfermedades sexualmente transmisibles. Los datos evidenciaram que los autores, en sua mayoria, son enfermros, actuan en el área de la docencia, son provenientes del Estado de São Paulo y han privilegiado la temática HIV! SIDA en detrimento de otras enfermedades sexualmente transmisibles, siendo su productión cientifica escasa y discontinua.

PALABRAS-CLAVE: Enfermedades sexualmente transmisibles. Sindrome de inmunodeficiencia adquirida. Publicaciones.

\footnotetext{
* Enfermeira junto ao HCFMRP-USP, Aluna de Pós-Graduação em Enfermagem, junto a Escola de Enfermagem de RibeirãoPreto -USE E-mail: renakari@zipmail.com.br

** Enfermeira, Professor Associado junto ao Departamento de Enfermagem Geral e Especializada da Escola de Enfermagem de Ribeirão Preto da Universidade de São Paulo. E-mail: egir@eerp.usp.br
} 


\section{INTRODUÇÃO}

Ao longo da história milenar das doenças sexualmente transmissiveis (DST), o estigma e o preconceito sempre se fizeram presentes, a ponto de se constituírem numa interferência negativa nas questões do seu enfrentamento.

Os profissionais da saúde através de ações educativas devem educar a população sobre a prevenção e os riscos das DST, bem como estimular a procura pelos Serviços de Saúde quando perceberem sintomas sugestivos de uma DST como corrimento, verrugas e feridas nos órgãos genitais. Estima-se que 12 milhões de novos casos de DST ocorram por ano no país, e destes, apenas $30 \%$ procuram os Serviços de Saúde e os demais $70 \%$ optam pela automedicação e/ou procuram por atendimento em farmácias ${ }^{(1)}$.

As DST sempre tiveram significado importante para a saúde pública, mas eram consideradas de maneira tímida e até subestimadas pelas autoridades de saúde. Com o surgimento da síndrome da imunodeficiência adquirida (aids), ficou evidente a sua relevância enquanto fator de risco para a mesma, e desta forma passaram a ser reconhecidas e valorizadas pelas autoridades de saúde, comunidade científica e população em geral(2)

A aids é a DST mais recente que se conhece e a única de notificação compulsória. Identificada no início da década de 80 , constitui-se num expressivo problema de saúde pública mundial. As pessoas portadoras do HIV/aids são vítimas de implicações físicas e biológicas devastadoras, bem como sociais, espirituais, psicoemocionais, e enfrentam sobretudo, o caráter estigmatizante de uma infecção que envolve a dimensão comportamental do indivíduo. A epidemia afeta todos os grupos sociais com predominância da faixa etária de $20 \mathrm{a}$ 39 anos, impondo diversos desafios à humanidade. Constitui-se objeto de constantes estudos e esforços, que buscam a prevenção e o controle da infecção/ doença, melhor assistência aos portadores e a descoberta de tratamentos e vacinas eficazes.

Dada a complexidade e a dinâmica da epidemia, o paciente requer assistência multi e interdisciplinar e atuação efetiva de uma equipe de enfermagem. Para essa assistência integral, as ações devem estar destituídas de atitudes preconceituosas e distorcidas e os profissionais devem estar continuamente atualizados.

A enfermagem tem papel fundamental no controle das DST/aids, seja desenvolvendo atividades de promoção e prevenção das mesmas intervindo individualmente, na familia ou na comunidade, ou detectando fatores e situações de risco, promovendo educação em saúde, contribuindo para o diagnóstico precoce, adesão e tratamento efetivo do paciente e seu parceiro sexual. Além disso, se responsabiliza para o acolhimento do paciente, seja prestando cuidado ou coordenando outros setores para a prestação da assistência(3)

Apesar dos avanços científicos, a doença é incurável e são alarmantes os números atuais da epidemia, tornando-se de grande importância o desenvolvimento da pesquisa, para que os conhecimentos gerados sejam divulgados e aplicados no ensino e na assistência, contribuindo desta forma, para um ensino de melhor qualidade e uma assistência mais humanizada e integral, visando melhorar também, a qualidade de vida dos portadores de HIV/aids.

Diante destas considerações torna-se relevante a realização de pesquisa sobre as DST/aids pois constituem como instrumento que produz conhecimento e que retroalimenta as atividades de ensino e assistência. Como profissionais que militam na área da saúde, temos constatado que o conhecimento disponível sobre aids é extenso, considerando-se o pouco tempo decorrido desde a sua identificação, ao mesmo tempo, temos nos deparado com a escassez de estudos de enfermagem quanto às demais DST.

Estudos analisando a produção literária em diversas áreas da enfermagem brasileira também apontam para escassez quantitativas de publicações por enfermeiros e ainda demonstram lacunas na construção do conhecimento, destacando a necessidade de maior envolvimento dos profissionais ${ }^{(4-5)}$

Conforme cita Tsunechiro, Carvalho, Posso, Elsas, Lui(6) a produção científica da enfermagem no Brasil preocupa os enfermeiros, diante da necessidade de estruturar o corpo de conhecimentos da enfermagem, principalmente na última década, visando melhorar a qualificação dos profissionais, torná-los mais atuantes na sociedade, aumentar seu campo de atuação e estabelecer vínculos com outros profissionais em equipes de trabalho.

Diante do exposto e da importância de se pesquisar sobre DST/aids realizamos este estudo que tem como objetivo geral, caracterizar os artigos sobre tais temáticas, em periódicos de enfermagem do Brasil, publicados no período de 1933 a 1999. Para tanto fizemos uma descrição sobre os autores quanto à titulação, formação profissional e procedência e sobre os artigos no que se refere ao tipo de DST abordada, aos conteúdos das publicações. Dessa forma buscamos apontar lacunas na produção científica acerca das DST/aids.

\section{MATERIAL E MÉTODOS}

Trata-se de um estudo exploratório, descritivo e documental acerca das temáticas DST/aids, baseamos na metodologia utilizada por Stefanelli(7). A população estudada constituiu-se de artigos publicados em 14 periódicos de enfermagem do Brasil, no período de 1933 
a 1999, quais sejam: Acta Paulista de Enfermagem (São Paulo-SP), Annaes de Enfermagem, Revista Baiana de Enfermagem, Revista Brasileira de Enfermagem (Brasília- DF), Revista Enfermagem Nova Dimensão, Revista Enfermagem Atual, Revista Enfermagem Moderna, Revista Enfermagem UERJ (Rio de Janeiro - RJ), Revista Enfoque (São Paulo SP), Revista da Escola de Enfermagem da USP (São Paulo - SP), Revista Gaúcha de Enfermagem (Porto Alegre - RS), Revista Latino Americana de Enfermagem (Ribeirão Preto -SP), Revista Paulista de Enfermagem (São Paulo-SP) e Revista Texto \& Contexto Enfermagem (Florianópolis-SC). Para a escolha do período do estudo e população adotamos alguns critérios, a seguir mencionados. Consideramos como período inicial o ano do primeiro artigo publicado em periódico nacional de enfermagem e o período final o ano cujo acesso aos periódicos estava viabilizado. Os periódicos foram selecionados pela sua especificidade e periodicidade. Para a coleta de dados, utilizamos o banco de dados LILACS, buscando os unitermos DST, HIV/Aids e enfermagem. Para análise dos artigos, utilizamos um roteiro contendo os seguintes itens: - caracterização do autor quanto à sua formação, área atuação e procedência; tipo de estudo realizado e temática abordada. Selecionamos os artigos com base na leitura dos títulos de todos os artigos contemplados nos periódicos selecionados, desde que contivessem a palavra DST/aids ou o nome de alguma DST explícito no título. Quando o título não apresentava as palavras-chave DST/aids, mas sugeria a abordagem de tais temáticas, procedemos à leitura do resumo dos trabalhos. Quanto aos artigos selecionados, estes foram lidos na íntegra e analisados pelo pesquisador.

Fizemos a apresentação dos dados através de tabelas, utilizando porcentagem simples e criação de categorias a partir de núcleos temáticos identificados.

\section{RESULTADOS E DISCUSSÃO}

No levantamento realizado selecionamos 73 artigos, dos quais 55 versavam exclusivamente sobre HIV/aids e 18 sobre as demais DST abrangendo artigos de pesquisa, de atualização, relatos de experiência, editoriais e notas prévias (Anexos I e II).

$\mathrm{Na}$ caracterização das publicações de acordo com o tipo de produção, a maior frequência $60(82,2 \%)$ foi de artigos de pesquisa; seguido de $8(19,9 \%)$ classificados como outros (editorial ou resenha) e de $5(6,8 \%)$ como relatos de experiências.

Embora o surgimento das DST, remonte à história da humanidade, não houve aumento significativo do número de publicações sobre esta temática nos periódicos de enfermagem ao longo dos anos. Observamos lacunas de publicações durante muitos anos. O primeiro artigo sobre DST localizado pelo pesquisador foi publicado na revista Annaes de Enfermagem em 1935, e somente trinta e sete anos depois, em 1972 encontramos o segundo artigo publicado.

Ao comparar o número de artigos a temática DST com HIV/aids fica evidente a prioridade que os autores que trabalham e pesquisam DST atribuem ao HIV/aids, desde a sua descoberta.

O primeiro artigo publicado encontrado sobre aids em periódicos nacionais de enfermagem data de 1986, quatro anos depois da notificação dos primeiros casos no Brasil e gradativamente todos os periódicos passaram a abordar esta temática, chegando a 10 artigos publicados em 1993.

Esse aumento no número de publicações sobre aids pode ser, devido ao seu caráter incurável, à rápida disseminação da doença que atinge milhões de pessoas em todo o mundo.

Quanto à formação profissional dos autores pesquisados, destaca-se o enfermeiro com $50(68,5 \%)$ dos artigos e em $10(13,7 \%)$ em co-autoria com outros profissionais, tais como médico, nutricionista, psicólogo, pedagogo e em um artigo está citado também, o técnico em enfermagem. Em oito artigos $(10,9 \%)$ houve a participação de alunos de graduação e pós-graduação. E em $5(6,8 \%)$ não foi especificado a formação profissional dos autores. O enfermeiro foi o profissional que mais publicou sobre tais temáticas nos periódicos pesquisados, o que era de se esperar dada a especificidade destes periódicos. Evidenciamos que é discreta a participação de outros profissionais da área da saúde e da educação pesquisando em conjunto com enfermeiros.

No entanto, deve ser estimulado o trabalho multiprofissional com a participação de profissionais de outras áreas do conhecimento a publicarem nos periódicos de enfermagem, mais ainda, que possam em co-autoria com enfermeiros contribuir com a ampliação do entendimento sobre as DST/aids, temas complexos que carecem de uma abordagem interdisciplinar.

Observou-se também, que há uma pequena participação de alunos de graduação e pós-graduação pesquisando as temáticas DST/aids, trabalho que deve ser estimulado visando à sensibilização para a problemática em questão.

Tanto na pesquisa quanto na assistência prestada aos indivíduos portadores do HIV/aids, é desejável que se efetive a atuação de diferentes profissionais, de maneira interdisciplinar, articulando as contribuições das diversas áreas do conhecimento na busca de um entendimento global para o enfrentamento da epidemia. Projetos interdisciplinares buscam compreender a complexa realidade da doença e abordar vários aspectos de origem biológica, social, psicológica, ética e legal(8). 
Em relação a área de atuação dos autores, verificamos que $38(52,0 \%)$ são docentes, $14(19,2 \%)$ são profissionais que atuam na assistência em coautoria com docentes e $8(10,9 \%)$ são alunos de graduação e pós-graduação que publicaram em parceria com docentes, e $7(9,6 \%)$ artigos não especificavam a área de atuação.

Apenas $5(6,8 \%)$ dos artigos foram publicados exclusivamente por profissionais assistenciais, e 1 $(1,4 \%)$ por alunos de graduação, o que evidencia que a preocupação de pesquisar e divulgar o conhecimento provém em sua maioria de profissionais envolvidos principalmente, com o ensino e pesquisa em enfermagem.

Estudos realizados por Oliveira, Persinotto(5) e Stefanelli(7) em outras áreas de investigação em enfermagem revelam dados semelhantes demonstrando o predomínio da docência sobre as demais áreas de atuação dos enfermeiros que publicaram em periódicos nacionais de enfermagem.

A participação dos profissionais assistenciais na construção do conhecimento em enfermagem, não se restringindo aos profissionais da área da acadêmica, é imprescindivel, para a implementação dos resultados das pesquisas na prática de enfermagem e busca de soluções de aspectos relevantes do cotidiano dos enfermeiros ${ }^{(9)}$

Entretanto, a falta de incentivo das instituições de saúde aos profissionais assistenciais no que se refere a produção científica, o número reduzido de periódicos nacionais indexados, somados às rígidas normas de publicação científica são fatores que podem dificultar realização de investigação científica por estes profissionais $(5,9)$

No que se refere à procedência dos autores (Tabela 1), a Região Sudeste destaca-se com o maior número de autores com publicações 49 (67,1\%), sendo que o Estado de São Paulo apresenta mais de $70 \%$ das publicações desta região. Em seguida vem a Região Sul com 19 $(13,7 \%)$ e Região Nordeste com $6(8,2 \%)$. Em 6 (8,2\%) publicações não foi possível caracterizar a origem regional e não encontramos nenhum autor da região Norte, conforme podemos observar na tabela 1 .

Tabela 1- Distribuição das publicações sobre DST/ aids em periódicos nacionais de enfermagem, segundo Região. Brasil, 1993-1999

\begin{tabular}{lcc}
\multicolumn{1}{c}{ Procedência } & Freqüência & $\%$ \\
\hline Sudeste: SP-39; RJ-8; MG-2 & 49 & 67,1 \\
Sul: SC-4; RS-4; PR-2 & 10 & 13,7 \\
Centro-Oeste: Brasilia-1; MT-1 & 2 & 2,7 \\
Nordeste: CE-3; RN-2; BA-1 & 6 & 8,2 \\
Norte: 0 & 0 & 0,0 \\
Não citada: 6 & 6 & 8,2 \\
\hline TOTAL & 73 & 100,0 \\
\hline
\end{tabular}

A região Sudeste contempla maior número de casos de aids desde o início da epidemia, além de ser também a que detém maior número de instituições de ensino superior e cursos de pós-graduação em enfermagem, características podem contribuir para maior volume de investigações científicas ${ }^{(10)}$

Entretanto, verificamos que os dados epidemiológicos atuais refletem a diversidade sociogeográfica da epidemia, demonstrando que existem casos de aids notificados em todos os Estados brasileiros. Observamos que até março de 2002, foram 237.588 os casos diagnosticados e notificados ao Ministério da Saúde, sendo que deste total 67,3\% (159.965 casos) são de residentes na região Sudeste, 16,4\% (39.028 casos) na região Sul, 9,4\% (22.249 casos) na região Nordeste, $5,1 \%$ (11.998) na região Centro-Oeste e 1,8\% (4.340 casos) na região Norte mil casos ficando a Região Sudeste com maior número de notificações, cerca de 142 mil; seguida pelas Regiões Sul com 29 mil e Nordeste com 18 mil casos(11).

Apesar do maior contigente de casos de aids estar concentrado nas Regiões Sul e Sudeste, observamos um aumento significativo do número de casos nas demais regiões brasileiras, principalmente nos primeiros anos da década de 90.

Além disso, mesmo estando a maior concentração de casos de aids do Brasil na Região Sudeste, observa-se uma desaceleração do crescimento da epidemia na população em geral e principalmente na população masculina dessa região, mostrando uma tendência à estabilidade. Porém, nas demais regiões, observamos aumentos relativos dos casos em municípios com menos de 50 mil habitantes, processo este chamado de interiorização. Baseados nesta constatação fica evidente que as ações de controle da epidemia é necessário o envolvimento efetivo dos profissionais de todos os Estados brasileiro, respeitando as características da epidemia em cada região.

Com referência à temática HIV/AIDS, são as seguintes as revistas que contemplam o maior número de publicações, no período estudado: Revista Brasileira de Enfermagem com 14 (26,4\%); Revista LatinoAmericana de Enfermagem, com $9(16,4 \%)$ e Revista da Escola de Enfermagem da USP, com 9 (16,4\%) artigos.

Em relação às demais DST, entre os periódicos com maior frequência de publicações, destacamos: Revista de Enfermagem da UERJ com 4 (22,2\%) artigos; Revista Paulista de Enfermagem com 3 $(16,7 \%)$, Revista Brasileira de Enfermagem com 2 $(11,1 \%)$, e os demais periódicos contemplaram apenas $1(5,5 \%)$ artigo. Destacamos que nenhum artigo sobre DST foi encontrado nos seguintes periódicos: Acta Paulista de Enfermagem, Revista Baiana de Enfermagem, Revista Enfermagem Nova Dimensão, 
Revista Enfermagem Atual, Revista Enfermagem Moderna, Revista Enfoque e Revista Texto \& Contexto Enfermagem.

Além do maior número de artigos tratarem sobre a aids, observou-se que há uma maior diversidade de autores, enquanto sobre as demais DST são os mesmos autores que publicam em periódicos diferentes.

Para identificarmos o enfoque principal dos 73 artigos analisados, procedemos à leitura dos artigos na integra, extraindo sua idéia central e agrupando-a em categorias, apresentadas na Tabela 2.

$\mathrm{Na}$ Tabela 2, observamos que na produção científica acerca de HIV/aids dos 55 artigos encontrados, os tópicos assistência de enfermagem e reações emocionais foram o foco central em 12 (21,82\%) e 6 $(10,9 \%)$ dos artigos encontrados respectivamente.

No conteúdo sobre assistência de enfermagem os pesquisadores se preocuparam em descrever as características desta doença de caráter transmissivel e estigmatizante, seus aspectos clínicos e epidemiológicos, principalmente nos primeiros artigos publicados. Trataram de questões de isolamento e prevenção de doenças. Com referência as reações emocionais os pesquisadores buscaram evidenciar o impacto psicossocial da doença. Buscaram compreender o significado de cuidar do paciente com aids, e de estar com aids. Apontaram o medo dos profissionais em cuidar destes individuos e evidenciaram sentimentos negativos provocados nos portadores do HIV/aids e sua família.

Neste estudo podemos observar em $5(9,1 \%)$ dos artigos a preocupação dos pesquisadores em investigar o conhecimento sobre o HIV/Aids em diferentes populações como trabalhadores rurais, manicures, estudantes universitários, e estudantes do nivel médio a fim de desenvolver projetos educativos voltados para estas populações. Em 5 (9,1\%) dos artigos os autores trataram sobre os dados e o perfil epidemiológico de determinadas populações ou instituições a fim de compreender o impacto da epidemia, sua dimensão e repercussão.

Identificamos que em outros $5(9,1 \%)$ dos artigos o enfoque foi sobre a utilização de medidas preventivas, incluídas nesta categoria as medidas de precaução padrão, e outras medidas de biossegurança relacionadas com a prevenção de acidentes.

Apesar dos vários aspectos abordados acerca do $\mathrm{HIV} /$ aids, sua gravidade e conseqüência constituemse no desafio contemporâneo para os profissionais da saúde e toda sociedade.

Identificamos que apesar do maior número de publicações se referirem a temática $\mathrm{HIV} /$ aids destacamos algumas temáticas relevantes que foram abordadas em 1 (1,81\%) artigo como associação HIV/ Tuberculose.
Com o aumento da sobrevida dos indivíduos acometidos pelo HIV/aids devido sobretudo aos avanços diagnósticos e terapêuticos, que caracteriza a aids enquanto doença crônica, destacamos que nenhum artigo abordou questões atuais da epidemia como qualidade de vida, adesão e tratamento com antiretrovirais. Questões sobre estilo de vida e comportamento de risco foram abordadas em 3 (5,45\%) dos artigos, entretanto, destacamos ausência de publicações que tratam exclusivamente sobre sexualidade.

De acordo com o Ministério da Saúde ${ }^{(11)}$ além de outras características observamos a feminilização em todas as regiões brasileiras, com maior inserção da mulher na epidemia(9) temática também abordada em apenas dois trabalhos.

Tabela 2. Distribuição dos conteúdos das publicações segundo temáticas DST e HIV/Aids. Brasil, 1933-1999.

\begin{tabular}{lrrr}
\hline Conteúdo & HIV / & DST & TO- \\
& Aids & & TAL \\
\hline Assistência de Enfermagem & 12 & 1 & 13 \\
Associação HIV/ Tuberculose & 1 & & 1 \\
Atitude profissional & 2 & & 2 \\
Aids e interdisciplinaridade & 1 & - & 1 \\
Conhecimento sobre Aids/DST & 5 & 1 & 6 \\
Comunicacãa/ Interação Verbal & 4 & - & 4 \\
Educação/ Ensino para & 4 & 8 & 12 \\
Programas Educativos & 3 & & 3 \\
Evolução Histórica / Brasil & 1 & & 1 \\
Prevalência/dados & 5 & 3 & 8 \\
Feminilizacão e Aids & 2 & & 2 \\
Reac̃̃es Emocionais & 6 & 1 & 7 \\
Estilo de vida/ Comportamento & 3 & 1 & 4 \\
de & 5 & & 5 \\
Medidas Preventivas / & & & \\
Auto-proteção & - & 3 & 3 \\
Risco/ ocorrência/ profilaxia & & 18 & 73 \\
\hline TOTAL & 55 & 18 \\
\hline
\end{tabular}

Em relação às demais DST observa-se de um modo geral a baixa produção científica. A pouca que existe concentra-se nas temáticas Educação/Ensino para prevenção das DST, com 8 (44,4\%) artigos; Prevalência/dados epidemiológicos e risco/ ocorrência e profilaxia com $3(16,7 \%)$, cada.

Encontramos apenas 1 artigo que tratou sobre assistência de enfermagem e reações emocionais na confirmação do diagnóstico de uma DST. Observamos lacunas na produção de conhecimento referente às DST, pois nos artigos analisados não encontramos nenhum trabalho que abordou programas educativos para a população em geral.

Estes mesmos artigos analisados relatam não sé a ausência de programas sistematizados de atenção às DST(12), como também a importância de programas educativos e sistematizados para a prevenção e controle das mesmas ${ }^{(13)}$

O estilo de vida e o comportamento de risco foi abordado em 1 (5,55\%) artigo, o que evidencia que 
questões sobre sexualidade foi pouco abordada por estes autores. Sabemos que a falta de educação sexual e a mudança comportamental ocorridas, principalmente no início da década de 70 com o uso de anticoncepcionais, maior número de parceiros sexuais, somados ao estima histórico vinculado às DST são fatores que dificultaram o controle das mesmas, e que contribuíram para o avanço da epidemia da aids(14).

Ressaltamos poucos trabalhos relatam experiências vivenciadas pelos enfermeiros tanto no âmbito hospitalar, ambulatorial ou na comunidade. Apesar de todo conhecimento produzido acerca destas doenças, acreditamos ser fundamental o envolvimento da enfermagem com a temática DST/aids de maneira interdisciplinar não apenas no que se refere a assistência mas também na produção do conhecimento. A realização e divulgação de trabalhos científicos por enfermeiros docentes e assistencias, é sem dúvida fundamental para o avanço do conhecimento científico na enfermagem.

A pesquisa retroalimenta o ensino e a assistência, configurando-se como básico instrumento destas interfaces. Desse modo a inter-relação desta tríade e deve ser enfatizada pelos profissionais em qualquer área temática, e principalmente quando se trata de problema de saúde coletiva, como é o caso das DST.

A magnitude e a transcendência das DST, a elevada incidência e prevalência, as altas taxas de complicações e as graves conseqüências psicossociais e econômicas incluem-nas no rol das doenças que merecem atenção e tratamento. Sem dúvida alguma, a conseqüência direta desta magnitude causa impacto sobretudo na saúde individual e coletiva, manifes-tando-se através de esterilidade, aborto, prematu-ridade, morbidade perinatal, mortalidade infantil, doenças neurológicas, entre outras.

Este panorama agrava-se ainda mais com os casos de infecção pelo papiloma vírus humano (HPV) na gênese do câncer cervical (15), na relação do vírus da hepatite $B$ com o câncer hepático e na inquestionável interação das DST com o HIV.

Por tudo isso, a atuação efetiva de uma equipe multiprofissional e interdisciplinar é imprescindivel na prevenção às DST, ressaltando-se a participação insubstituivel da enfermagem e a importância da pesquisa enquanto elemento que retroalimenta o ensino e a assistência.

\section{CONSIDERAÇÕES FINAIS}

As DST ao longo da história, sempre representaram expressivo problema de saúde pública, frente a sua alta incidência e dificuldade para tratamento adequado e prevenção, e com o advento da aids ficou acentuada a preocupação das autoridades governamentais com seu controle.
Os resultados apresentados neste estudo evidenciam que durante muitos anos, artigos que abordavam exclusivamente as DST eram escassos e descontínuo em periódicos nacionais de enfermagem, porém com o advento da aids, os pesquisadores demonstraram grande preocupação com essa problemática, principalmente porque se tratava de uma doença nova transmissivel e de caráter letal.

Observamos um "salto" nas publicações referentes ao HIV/aids, a partir da década de 80, com predomínio de artigos sobre esse tema. Não podemos negar a importância e a preocupação da epidemia da aids no cenário nacional e mundial, tendo em vista a sua magnitude e rápida disseminação, a qual atinge milhões de pessoas em todo mundo; entretanto, as DST remontam à história da humanidade, e sabemos que grande parte dos indivíduos doentes não procuram assistência médica adequada.

Este dado é preocupante, pois mesmo diante de tratamentos eficazes contra tais doenças, quando não diagnosticadas e tratadas precocemente, elas podem evoluir para complicações graves, além de se constituírem num fator facilitador para a aquisição do HIV.

No presente estudo os autores, em sua maioria, são enfermeiros e atuam como docentes em instituições de ensino em enfermagem. As publicações sobre HIV/ aids contam com uma participação pequena de enfermeiros assistenciais em parceria com docentes, como também é discreto o envolvimento de outros profissionais e alunos de graduação e pós-graduação na produção científica.

Os resultados apresentados evidenciam que a produção científica concentra-se na região Sudeste, nos Estados de São Paulo e Rio de Janeiro, e que há necessidade de maior produção e divulgação de forma mais generalizada das temáticas DST/aids.

A partir de sua descoberta, a aids foi a DST mais abordada e por uma diversidade de autores, diferentemente das publicações encontradas quanto às demais DST.

Sendo estas doenças um desafio contemporâneo, consideramos de fundamental importância a sensibilização e o envolvimento dos profissionais de maneira interdisciplinar, tanto na pesquisa como na assistência. A estratégia mais efetiva para atuar junto às DST/aids é através da prevenção e do controle, por meio de constante atualização e sensibilização dos profissionais da saúde e da população em geral. A divulgação das mais variadas experiências educativas devem ser estimuladas, abordagens que contemplem a percepção de risco, mudanças no comportamento sexual e a adoção de comportamentos seguros. O envolvimento de enfermeiros pode contribuir para detecção de situações de risco e educação em saúde dos indivíduos portadores de uma DST e também de seus parceiros sexuais ${ }^{(2)}$. 


\section{REFERÊNCIAS BIBLIOGRÁFICAS}

(1) Gir E, Moriya TM, Costa JC, Duarte G, Oliveira MHP, Bueno SMV, Tavares MSG. Estudo das condutas adotadas por balconistas de farmácias frente a casos relatados de gonorréia. Rev Med 1991; 24 (1):15-25.

(2) Ministério da Saúde. Coordenação Nacional de DST/AIDS/ Manual de controle das Doenças Sexualmente Transmissiveis (DST). $3^{\mathrm{a}}$ ed. Brasilia, 1999.

(3) Rocha SMM, Almeida, MCP. O processo de trabalho da enfermagem em saúde coletiva e a interdisciplinaridade. Rev Lat -Am Enferm 2000; 8 (6): 96-101.

(4) Telles PCPF, Cassiani SHB. O "Estado da Arte" da informática em Enfermagem: revisão de periódicos. Rev Baiana Enferm 1998; 11(2): 38-49.

(5) Oliveira SMJV, Persinotto MOA. Revisão de literatura em Enfermagem sobre hipertensão arterial na gravidez. Rev Esc Enferm USP 2001; 35(3):214-22.

(6) Tsunechiro MA, Carvalho DV, Posso MBS, Elsas BX, Lui MC, Stefanelli MC. Instrumento para análise de periódicos de enfermagem. Rev Esc Enferm USP 1983; 17(2): 107-17.

(7) Stefanelli MC, Carvalho DV, Elsas BX, Tsunechiro MA, Posso MBS, Lui MC. Análise da Revista da Escola de Enfermagem da USP no período de 1967 a 1981. Rev Esc Enferm USP 1987; 21(3):263-284.
(8) Xavier IM, Leite JL, Tocantins, FR. Aids como campo de pesquisa interdisciplinar e multiprofissional. Rev Bras Enferm 1993; 46 (3/4): 309-313.

(9) Lopes, CM. Produção de conhecimentos por enfermeiros assistenciais: sua utilização na prática. [Tese] Ribeirão Preto (SP): Escola de Enfermagem de Ribeirão Preto/USP; 1990.

(10)Luis MAV, Nogueira MS, Diniz SA, Oliveira, ER. Uma amostra da produção científica da região Sudeste do Brasil no período de 1987-1991. Rev Lat Am Enferm 1993; 1(nºsp): 69-83.

(11) Ministério da Saúde. Boletim Epidemiológico AIDS. 2001/ 2002.

(12) Mendes IJM, Moriya TM, Gir E, Oliveira MHP, Tavares MS, Bueno SMV. Sífilis e Gonorréia na cidade de Ribeirão Preto. Enfoque 1989; 17 (2):45-47.

(13) Gir E, Moriya TM, Robazzi MLCC. Ações educativas em doenças sexualmente transmissiveis. Rev Gaúcha Enferm $1991 ; 12(1): 38$.

(14) Oliveira MHP, Vietta EP, Moriya TM, Gir E. Reações emocionais dos portadores de doenças sexualmente transmissiveis no momento da confirmação do seu diagnóstico. Rev Bras Enferm 1987; $40(1): 38-42$.

(15)Monsonego J, Dargent D, Naud P Infecção pelo Papilomavírus Humano. In: Naud P Doenças sexualmente transmissiveis \& Aids. Porto Alegre: Artes Médicas; 1993. p.118-132.

\section{ANEXO I \\ ARTIGOS SOBRE DST QUE CONSTITUIRAM A AMOSTRA DO. ESTUDO}

1- Aloi AP, Souza RC, Cabrita KST. Orientação dos adolescentes sobre DST em consulta de enfermagem. Rev Enferm UERJ 1994; $2(1): 126$.

2- Barros SMO, Ruggero EMS. Prevalência da soropositividade para a Sífilis entre algumas gestantes no Serviço Pré-Natal do Amparo Maternal. Rev Paul Enferm 1994;13(1/3):28-31.

3- Cruz ICF. Carnaval e prevenção da Aids/DST em mulheres: a enfermagem atuando (Bem!) em espaços não convencionais. Rev Enferm UERJ 1995; 3(1):121-3

4- Gir E, Moriya T.M, Duarte G, Machado AA. Medidas preventivas contra aids e outras DST conhecidas por universitários da área da saúde. Rev Lat Amer Enferm 1999; 7(1):11-7.

5- Gir E, Moriya TM, Oliveira MHP, Mendes IJM, Tavares MSG. Levantamento retrospectivo de algumas DST. Rev Paul Enferm $1990 ; 9(1): 3-7$.

6- Gir E, Moriya TM, Oliveira MHP, Pelá NTR. Informação/Educação em DST/AIDS/Sexualidade Humana - um relato de experiência de 14 anos. Rev Esc Enferm USP, 1998; 32(4):291-6.

7- Gir E, Moriya TM, Robazzi MLCC. Ações educativas em Doenças Sexualmente Transmissíveis. Rev Gaúcha Enferm 1991; 12(1):38-40.

8- Leone M. Siflis. Annaes de Enferm 1935; 3(6): 26.

9- Margarido SA. Estudo sobre doenças sexualmente transmissiveis em estudantes universitários. Rev Esc Enferm USP 1980; $14(2): 201$ 
10- Margarido SA. Situação do ensino das Doenças Sexualmente Transmissiveis nos cursos de graduação em Enfermagem do município de São Paulo. Rev Esc Enferm USP 1981; 5(3):275-88.

11- Mendes MJI, Moriya TM, Gir E, Oliveira MHP, Tavares MSG, Bueno SMV. Sífilis e gonorréia na cidade de Ribeirão Preto-SP Enfoque 1989; 17(2): 45-7.

12- Moriya, TM, Tavares MSG, Oliveira MHP, Gir, E. O que os jovens pensam sobre doenças sexualmente transmitidas? Rev Bras Enferm 1985; 38(3/4):300-5.

13- Oliveira MHP, Vietta EP, Moriya TM, GIR E. Reações emocionais dos portadores de doenças sexualmente transmissiveis no momento da confirmação do seu diagnóstico. Rev Bras Enferm 1987; 40(314):38-42.

14- Silva MTN. DST: um desejo para a juventude. Rev Enferm UERJ 1995; 3(1):114.

15- Tsunechiro MA. Estudo sobre o risco e a ocorrência da infecção ocular gonocócica em 112 recém-nascidos. Rev Esc Enferm USP 1979; 13(1): 95.

16- Tsunechiro MA. Revisão bibliográfica sobre a profilaxia da conjuntivite gonocócica no recém-nascido. Rev Esc Enferm USP 1979; 13(3):275-86.

17- Valle MDB. Abordagem sindrômica das DST. Rev Enferm UERJ 1995; 3(1):121-3.

18- Vinha, VHP, Almeida MCP, Volpi CLO. Sífilis - orientação e assistência de Enfermagem de saúde pública num hospital escola. Rev Bras Enferm 1972; 15(1/2):70-80.

\section{ANEXO II}

\section{ARTIGOS SOBRE HIV/AIDS QUE CONSTITUÍRAM AMOSTRA DO ESTUDO}

1- Barros SMO, Levantezi M. Comportamentos de risco para infecção pelo HIV relatados por gestantes atendidas no serviço de prénatal do Amparo Maternal. Rev Paul Enferm 1993; 12(3):122-5.

2- Barros SMO, Marin H, Myazawa N, Cozzupoli CA. Cuidados domiciliares ao paciente com síndrome da imunodeficiência adquirida. Acta Paul Enferm 1989; 2(1): 30-2.

3- Barroso MGT, Miranda CCL, Pinheiro PN C. A aids sob o olhar da companheira contaminada. Rev Bras Enferm 1998; 51(3):393-402.

4- Bergamo M, Marcelino K, Garrido LS. Procedimentos Técnicos Básicos na prevenção da aids num centro cirúrgico de grande porte. Rev Bras Enferm 1988; 41(2):155-60.

5- Carvalho RO. Aids e a escola: proposta interdisciplinar e institucional de prevenção. Rev. Bras Enferm 1998; 51(2):245-52.

6- Chida AM, Arantes DV, Meneghin P, Cianciarullo TI. Dia mundial da Aids na Escola de Enfermagem da Universidade de São Paulo. Rev Paul Enferm 1990; 9(1):36.

7- Dessunti EM. Percepções de discentes e equipe de enfermagem em relação ao paciente com suspeita ou diagnóstico confirmado de aids. Rev Gaúcha Enferm 1989; 10(1):45-51.

8- Dessunti EM, Soubhia Z. Perfil epidemiológico da aids no Estado do Paraná e região de Londrina - uma proposta para a assistência de enfermagem. Rev :Bras Enferm 1991; 44(2-3):103-12.

9- Dresler DE, Boemer MR. O ser com Aids em sua dimensão existencial. Rev Bras Enferm 1993; 46(1):7-20.

10- Dresler DE, Boemer, MR. O significado do cuidado do paciente com aids: uma perspectiva de compreensão. Rev Bras Enferm 1991; 44(1):70-81.

11- Dreher ML, Ferreira SRS, Eidt OR. Prevenção da Aids: experiência participativa com adolescentes de uma escola estadual de $1^{9}$ grau em Porto Alegre. Rev Gaúcha Enferm 1995; 16(1/2):52-7.

12- Figueiredo, M.A, Morais KC. Aids e enfermagem: atitudes versus traços da personalidade no contexto do atendimento hospitalar. Rev Lat Am Enferm 1994; 2(1):41-56.

13- Gabriel R, Fakih FT. Proposta de ficha cadastral e banco de dados informatizado para levantamento de dados epidemiológicos e de evolução clínica dos clientes soropositivos para o HIV.. Rev Bras Enferm 1993; 46(3/4):317-26. 
14 - Gir E, Costa FPP, Silva AM. A enfermagem frente a acidentes de trabalho com material potencialmente contaminado na era do HIV Rev Esc Enferm USP 1998; 32(3):262-72

15 - Gir E, Gessolo F. Conhecimentos sobre aids e alterações nas ações profissionais das manicures de Ribeirão Preto. Rev Esc Enferm USP 1998; 32(2):91-100.

16 - Gir E, Moriya TM, Figueiredo MAC, Duarte G, Carvalho MJ. Avaliação dos riscos da infecção pelo HIV segundo diferentes práticas sexuais na perspectiva de estudantes universitários e especialistas em HIV/AIDS. Rev Esc Enferm USP 1999; $33(1): 416$.

17 - E, Moriya TM. Interação verbal entre a equipe de enfermagem e o paciente aidético. Rev Esc Enferm USP 1990; $24(2): 30113$.

18 - Gir E, Moriya TM. O estudo comparativo sobre a interação verbal entre enfermeiras e atendentes de enfermagem com o paciente portador de Aids. Rev Esc Enferm USP 1993; 27(3):387-402.

19 - Gir E, Moriya TM, Dela Coleta JA. Teoria da informação: uma altermativa metodológica o estudo da interação verbal enfermeiro/paciente aidético. Rev Gaúcha Enferm 1993; 14(2):107-16.

20 - Gir E, Moriya TM, Dela Coleta JA, Stephaneck P, Pelá NTR. Teoria da informação como instrumento de pesquisa na integração verbal entre o portador de aids e a equipe de enfermagem. Rev Esc Enferm USP 1993; 27(1):102-7.

21 - Kohlrausch E, Sensibilizando as enfermeiras para o cuidado transpessoal no atendimento aos portadores do HIV e doentes de AIDS: relato de experiência. Rev Gaúcha Enferm 1999; 20(1):120-31.

22 - Kusano MSE. Estudo comparativo entre tuberculosos não infectados e infectados pelo HIV, no Distrito Federal. Rev Bras Enferm 1996; 49(1):41-54.

23 - Lopes GT, Spindola T. Sentimentos de mães de portadores de aids. Rev Enferm UERJ 1994; 2(2):183-91.

24 - Lopes MVO, Fraga MNO. Pessoas vivendo com HIV: estresse e suas forma de enfrentamento. Rev Lat Am Enferm 1998; 6(4):75-81.

25 - Margarido SA. Contribuição para a participação do enfermeiro nos programas de prevenção e controle da aids. Enfoque 1988; 16(3):61-4.

26 - Meneghin P Aids: assistência de enfermagem e revisão de literatura. Rev Paul Enferm 1986; 6(3):99-107.

27 - Meneghin P. Entre o medo da contaminação pelo HIV e as representações simbólicas da Aids: o espectro do desespero contemporâneo. Rev Esc Enferm USP 1996; 30(3): 399-415.

28 - Moriya TM, Gir E, Hayashida M. Escala de atitude frente à aids: uma análise psicométrica. Rev Lat Am Enferm $1994 ; 2(2): 37-53$.

29 - Nichiata LYI, Shima H, Takahashi RE Buscando a compreensão do enfrentamento da aids no Brasil. Rev Lat Am Enferm 1995; 3(1):149-58.

30 - Oliveira AD, Vigas CRS, Sabka E, Guerra M, Baltazar R. Conhecimentos sobre prevenção da SIDA entre profissionais e acadêmicos da área da saúde. Rev Gaúcha Enferm 1996; 17(2):124-31.

31 - Oliveira MHP, Gir E, Moriya TM, Bueno SMV, Boreli OC. O conhecimento sobre aids entre trabalhadores rurais, Rev Paul Enferm 1992; 1(2): 77-80.

32 - Oliveira SMJV, Yoshioca MR. Subsídios para os profissionais de enfermagem em serviços de maternidade frente a problemática da Aids. Enfoque 1989; 17(4):101-5.

33 - Paiva MS. A feminilização da aids: uma questão de gênero? Rev Bras Enferm 1999; 52(1):7-17.

34 - Praça NS. Precauções universais na assistência à mulher no ciclo gravídico puerperal. Rev Paul Enferm 1995; 14(1):3-5.

35 - Praça NS, Grandi JL. A enfermeira obstétrica e a prevenção no risco ocupacional de contaminação pelo virus HIV em centro obstétrico. Rev Bras Enferm 1989; 42(1/4):101-5.

36 - Pedrazzani ES, Pires MA, Gouveia HG. Aids: estudo da epidemia na cidade de São Carlos. Rev Bras Enferm 1995; 48(2):120-6.

37 - Repetto M A, Souza MF. Congruência entre o enfermeiro e o paciente com síndrome da imunodeficiência adquirida quanto aos conceitos: percepção da doença e imagem corporal. Acta Paul Enferm 1999; 12(2):85-93. 
38 - Rodrigues AB, Magalhães MBB, Sales SMM. A questão do vírus da imunodeficiência humana e auto-proteção no trabalho. Rev Bras Enferm 1995; 48(3): 272-85.

39 - Rossi MJ, Meneghin P, Arantes AV, Munaro J. O papel da enfermagem no tratamento e prevenção da Aids. Enfoque 1987; 15(n1):19-20.

40 - Santos IMM, Matos MAB, Santos MF, Silva M. Perfil da aids em indivíduos acima de 50 anos. Rev Texto \& Contexto Enferm $1997 ; 6(2): 345-51$

41 - Shima H, Afusp DS, Nichiata LYI. Aids entre a população nipo-brasileira residente no município de São Paulo: dimensão e perfil do grupo estudado. Rev Esc Enferm USP 1998; 32(4):354-61.

41-Silva AL. A música no processo de cuidar de clientes com síndrome neurológica decorrente da aids. Rev Bras Enferm 1993; 46(2):107-16.

42 - Silva AL. O processo de cuidar de clientes com Aids, subsidiado pelo modelo homeodinâmico. Rev Gaúcha Enferm 1993; $14(2): 73-80$

43 - Silva AL. Reflexões sobre a experiência de uma. enfermeira no cuidar de clientes com aids, com base no modelo homeodinâmico. Rev Gaúcha Enferm 1993; 14(2):94-101.

44 - Spindola T, Alves CF. Perfil de mulheres portadoras do HIV de uma maternidade no Rio de Janeiro. Rev Esc Enferm USP 1999; 33(1):66-80.

45 - Takahashi RF, Shima H, Souza M. Mulher e aids: perfil de uma população infectada e reflexões sobre suas implicações sociais. Rev Lat Am Enferm 1998; 6(5):59-65.

46 -Torres GV, Davin RMB, Almeida MCS. Conhecimento e opiniões de um grupo de adolescentes sobre a prevenção da aids. Rev Lat Am Enferm 1999; 7(2):41-6.

47 - Torres GV, Enders BC. Atividades educativas na prevenção da aids em uma rede básica municipal de saúde: participação do enfermeiro. Rev Lat Am Enferm 1999; 7(2):71-7.

48 - Vega MS, Teixeira AB, Oliveira M. Atendimento a pacientes com sindrome da imunodeficiência adquirida em regime de hospital dia: relato de experiência. Rev Gaúcha Enferm 1998; 199(2):142-6.

49 - Vieira AAB. Assistência de enfermagem na Aids. Enfoque 1987; 15(3):67-70.

50 - Xavier IM. Cidadania, gênero e saúde: a mulher e o enfrentamento da Aids. Rev Enferm UERJ 1996; extra:89-100.

51 - Xavier IM, Leite JL, Braga GM, Nunes PHS. Enfermagem e aids: saber e paradigma. Rev Lat Am Enferm 1997; 5(1):65-73.

52 - Xavier IM, Leite JL, Nunes PHS, Braga GM. Aids: paradigma emergente. Rev Enferm UERJ 1994; 2(2): $198-205$.

53 - Xavier IM, Leita JL, Tocantins FR. Aids como campo de pesquisa interdisciplinar e multiprofissional. Rev Bras Enferm 1993; 46(3/4):309-13.

54 - Westrupp MHB, Pimentel CPB, Coelho EBS, Caetano JC, Souza AN. Educando para a saúde- conhecimento e fonte de informação sobre sindrome da imunodeficiência humana adquirida (SIDA). Rev Lat Am Enferm 1996; 4(2):61-71.

\section{" Artigo recebido em 18/06/01

